\section{Nulling Signal of a Slice, Slice Select Profile, and Radiofrequency Power}

\section{OVERVIEW}

In UNIT B2.2, we discussed the basic concept of RF excitation of a set of spins in a given volume. An RF pulse is initially applied to rotate the spins toward the transverse plane. In the presence of a slice select gradient, only certain spins, those within the slice of interest, are tipped by the RF pulse. These spins generate the MR signal. After the data are collected, but before the next RF pulse is applied, some signal from these spins may still remain. In order not to mix this leftover signal with the signal generated from the next RF pulse, a dephasing gradient is used before the beginning of the next RF pulse. In Fig. B2.3.1, the shaded areas $A$ and $B$ represent such dephasing gradients. The detailed calculations of the timings shown in Fig. B2.3.1 are presented in the Technical Discussion section.

Ideally, the transverse magnetization is proportional to the sine of the flip angle. However, because the RF transmit and receive coils are not perfect, it is difficult to say a priori what average flip angle will generate a maximum signal (see Fig. B2.3.2, panel A). This process is further complicated because the signal is integrated over the excited slice or volume. To find the maximum, the signal response can be adjusted by calibrating the current through the RF pulse. The current which gives the maximum signal will reflect an effective flip angle of $90^{\circ}$.

When the RF transmit coil or RF pulse design is not perfect, it is necessary to determine the profile of the excited slice. After rephasing the spins excited by the RF pulse in the presence of the slice select gradient, another gradient is turned on to continue to dephase the spins. Then, a read gradient is placed along the slice select direction to rephase the spins, and thus an echo is formed during the sampling window (see Fig. B2.3.3). The reconstructed image from these data represents the profile of the slice (integrated over the excited plane).

When the flip angle is small, the slice profile is a perfect boxcar shape. This is because the profile is the inverse Fourier transform of the RF field in this case (see UNIT B2.2). When the RF pulse is a sinc function, its inverse Fourier transform is a boxcar (rect) function (Fig. B2.3.4). If the RF field is Gaussian, then the profile is Gaussian as well. In general, the slice profile is similar to the shape illustrated in Figure B2.3.5. Because the slice profile is not a boxcar shape, usually the FWHM (full width at half maximum) is used to define the slice thickness. However, for a Gaussian pulse, FWTM (full width at tenth maximum) is more suitable to use.

The radiation power from the RF pulse is limited by the Food and Drug Administration (FDA) rule: $3 \mathrm{~W} / \mathrm{kg}$ over the body weight, or $8 \mathrm{~W} / \mathrm{kg}$ locally. That means, one cannot design an arbitrary RF pulse that requires too much power. Generally speaking, the radiation energy is proportional to the Larmor frequency, the square of the RF field, and the time duration of the RF pulse (or the inverse of the RF bandwidth). As one may recall from UNIT B1.2, the flip angle is proportional to the RF field and the duration of the RF pulse. Thus, the radiated energy is proportional to the square of the flip angle and the RF bandwidth. The usual RF bandwidth is about $2 \mathrm{kHz}$. In Figure B2.3.6, we list three different RF pulses, their flip angles at the center of the slice, and their radiation energies.
Radiofrequency Excitation and

Reception

B2.3.1

Supplement 14 


\section{TECHNICAL DISCUSSION}

In this unit, we discuss three technical topics: how to dephase spins prior to applying an RF pulse, how to image the slice profile itself, and the power radiated from certain RF pulses.

\section{Implementing Slice Selection}

Slices are excited by applying an RF pulse in the presence of a gradient. However, instantaneously pulsed, square-gradient waveforms are not achievable in practice. In this section, symmetrical trapezoidal gradient waveforms are considered as models of realistic gradient profiles with nonzero ramp-up and ramp-down times. The sequence diagram for slice selection is shown in Fig. B2.3.1.

It is assumed that all transverse magnetization is zero prior to the start of the RF excitation, but, as a design measure, $G_{\mathrm{ss}}$ is frequently applied prior to starting the RF pulse, to dephase any remnant transverse magnetization as well as to stabilize the gradient. The gradient area between $t_{0}$ and $t_{2}$ (area $A+B$ in Fig. B2.3.1) is adjusted to dephase all spins across a rectangular selected slice centered at $z_{0}$ :

$$
\int_{z_{0}-\frac{\mathrm{TH}}{2}}^{z_{z_{0}+\frac{\mathrm{TH}}{2}}^{2}} d z e^{i \phi(z)}=0
$$

where TH is the slice thickness, and it is assumed that the spins in the slice all have the same initial phase. The phase $\phi(z)$ is accumulated by spins at position $z$ during the time between $t_{0}$ and $t_{2}$, and is linear in $z$ :

$$
\phi(z)=-\gamma z \int_{t_{0}}^{t_{2}} d t G_{\mathrm{ss}}(t)
$$

The dephasing constraint Equation B2.3.1 will be satisfied if the difference $\phi\left(z_{0}+\mathrm{TH} / 2\right)$ $-\phi\left(z_{0}-\mathrm{TH} / 2\right)$ is a nonzero integer multiple of $2 \pi$. The minimum area to accomplish this is thereby found to satisfy:

$$
\gamma(A+B) \mathrm{TH}=2 \pi
$$

Typically, since $G_{0 z}$ is fixed by the slice selection process, Equation B2.3.2 is used to compute $t_{2}-t_{0}$. For example, if $t_{1}-t_{0}$ is $0.6 \mathrm{msec}$, TH is $2 \mathrm{~mm}$, and $G_{0 \mathrm{z}}$ is $23.49 \mathrm{mT} / \mathrm{m}$, then $t_{2}-t_{0}$ is $0.8 \mathrm{msec}$.

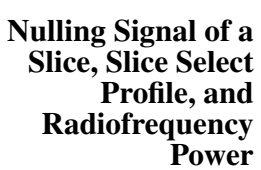

Power

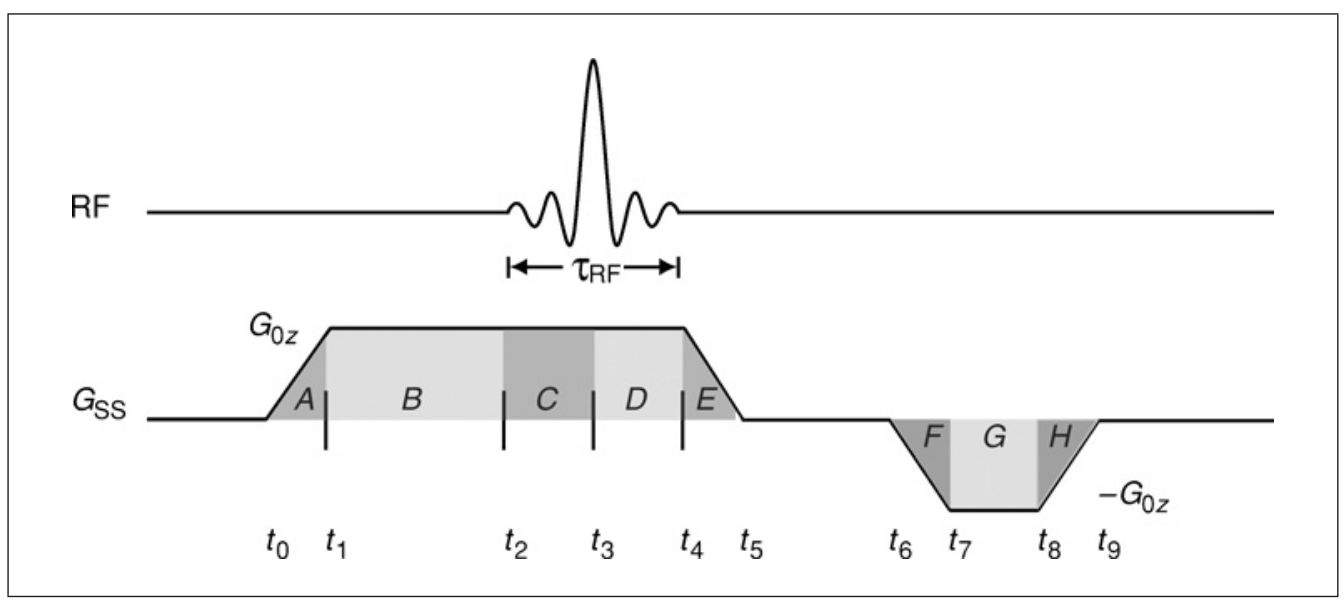

Figure B2.3.1 Sequence diagram for the trapezoidal-gradient slice selection. 
The RF pulse is assumed to be a truncated sinc pulse, and starts at $t=t_{2}$ and ends at $t=t_{4}$. After the RF pulse is turned off, the positive lobe is ramped down to zero and a negative lobe (also of trapezoidal shape) is applied to rephase the spins. (The reader may recall from UNIT B2.2 that a rephasing gradient is necessary after the selective RF pulse.) For small flip angles, the cancellation of gradient area to rephase the spins requires

$$
D+E=F+G+H
$$

in terms of the areas in Fig. B2.3.1. For a $90^{\circ}$ flip angle, the area $F+G+H$ will slightly larger than the area $D+E$ (see UNIT B2.2). If the rephasing lobe is symmetric and has the same magnitude $\left(G_{0 z}\right)$ for its plateau amplitude, Equation B2.3.4 restricts the timings of Figure B2.3.1 according to:

$$
\left(t_{4}-t_{3}\right)+\frac{1}{2}\left(t_{5}-t_{4}\right)=t_{8}-t_{6}
$$

since $t_{9}-t_{8}=t_{7}-t_{6}$. Note also that the RF pulse has been assumed to be symmetric about its center, which occurs at time $t_{3}$, so the gradient area from $t_{2}$ to $t_{3}$ is equal to the area between $t_{3}$ and $t_{4}$.

For a slice thickness $\mathrm{TH}=2 \mathrm{~mm}, G_{0 z}=23.49 \mathrm{mT} / \mathrm{m}$, and a rise time of $0.6 \mathrm{msec}$, the bandwidth of the RF pulse is

$$
\begin{aligned}
B W_{\mathrm{RF}} & =\psi \cdot \mathrm{TH} \cdot \mathrm{G}_{0 \mathrm{z}} \\
& =2 \mathrm{kHz}
\end{aligned}
$$

Consider a specific imaging example with a low flip angle. Take $\tau_{\mathrm{RF}}=t_{4}-t_{2}=5.12 \mathrm{msec}$ and let $t_{6}=t_{5}$ (the negative lobe immediately follows the positive lobe). The first zero crossing of the sinc pulse used to generate the slice profile in this case occurs at a time $t_{z c}=1 / B W_{\mathrm{RF}}$ from the center of the RF pulse, or $0.5 \mathrm{msec}$. This gives the number of zero crossings in $\tau_{\mathrm{RF}}$ to be $\mathrm{n}_{\mathrm{zc}}=10$ which is usually considered good enough for obtaining well-defined slice profiles, especially after apodizing the RF pulse (see UNIT B2.2).

\section{Calibrating the RF Field}

When a body is loaded into the RF coil, the amplitude of the RF pulse must be varied until the flip angle reaches a specific value, usually chosen to be $90^{\circ}$ or $180^{\circ}$ (see Fig. B2.3.2, panel B). For example, when the RF pulse creates an angle $\theta$ which goes through $90^{\circ}$, the transverse magnetization (and, hence, the measured signal) reaches a maximum; when $\theta$ goes through $180^{\circ}$, the transverse magnetization passes through zero (Fig. B2.3.2, panel A). For the signal maximum to occur at $\theta=90^{\circ}$ and the correct calibration to be done, the repeat time of the experiment must be large compared to the longitudinal relaxation times of the sample $T_{R} \gg T_{1}$. Usually a finite slice is excited and the RF field is assumed to be uniform over this slice.

An initial approach to calibrate the RF amplitude might be to find the maximum of the signal and associate this with a $90^{\circ}$-pulse. The problem with this approach is choosing a maximum value for the transverse magnetization $\left(M_{\perp}\right)$ in the presence of noise. Fitting $M_{\perp}(\theta)$ to $\sin \theta$ helps to reduce the errors due to noise. It is much easier to find the signal zero near $180^{\circ}$ where the slope $M_{\perp}(\theta)$ is the largest (see Fig. B2.3.2, panel A). The value for $B_{1}$ is then chosen for a given $\theta$ relative to this value. Actually, since the RF transmitter has a nonlinear response, a calibration curve must be made to obtain accurate values of $\theta$ as a function of the input power to the system.

Radiofrequency Excitation and Reception

B2.3.3 


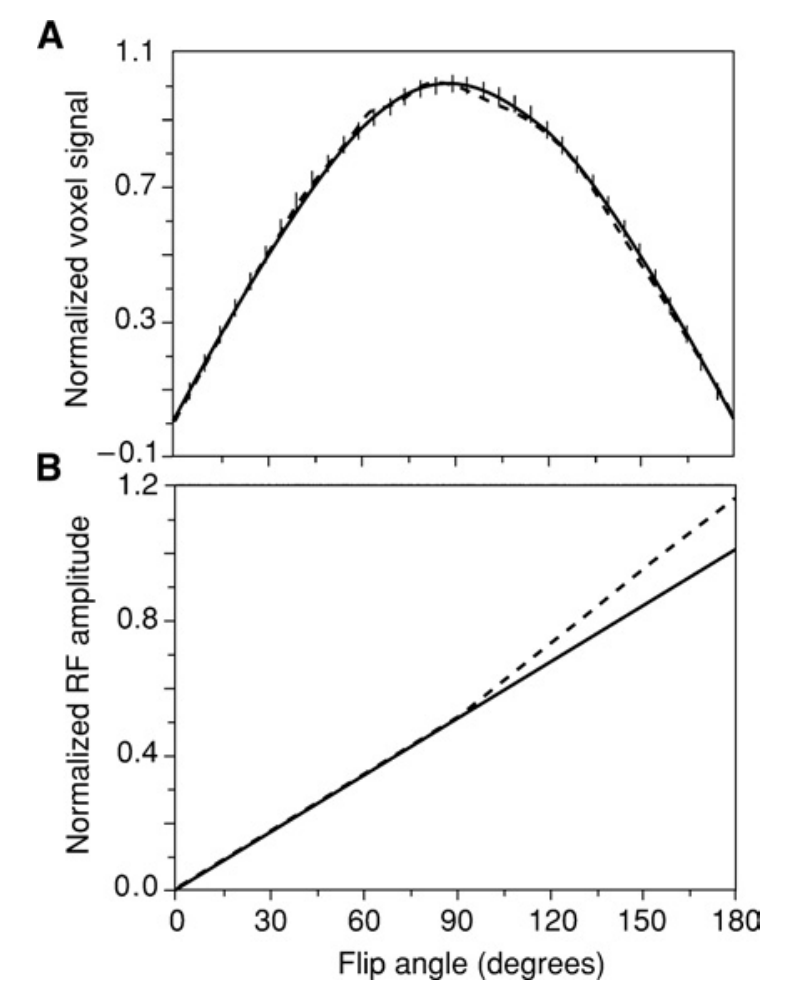

Figure B2.3.2 (A) $M_{\perp}$ as a function of $\theta$ with associated error bars during measurement of this function. The dashed line in panel A represents the measurements, and the solid line represents the least squares fit to the data. Error bars in the plot represent \pm 2 noise standard deviations. (B) Plot of the ideal current increase (solid line) and the current amplitude (dashed line) required because of transmitter nonlinearities to generate the correct $\theta$-pulse as a function of $\theta$.

For transmit coils which are inhomogeneous, the choice of which $B_{1}$ amplitude applied by the system gives a zero signal will change as the object size and spin density change. For example, if $B_{1}{ }^{\text {transmit }}$ is homogeneous only over roughly the central half of its volume, then for a small object in the center of the coil, $B_{1}$ associated with the $180^{\circ}$-pulse (referred to as $\left.B_{1,180}\right)$ will be correct everywhere. If the object is large compared to the uniform region of the coil, then $B_{1}=B_{1,180}$ will not necessarily generate a zero signal. This is due to the fact that for spins not at the coil center, $B_{1}$ at those positions will create an angle greater than (or less than) $180^{\circ}$ even when $B_{1}$ at the center equals $B_{1,180}$. Since the pick-up coil receives an integrated signal, it will take a field at the center smaller than (or greater than) $B_{1,180}$ to generate a $180^{\circ}$-pulse in the outer regions of the phantom. The measured value of $180^{\circ}$ will occur when the volume integral of the transverse magnetization is zero.

\section{Checking the $R F$ profile}

It is also necessary to measure the effective slice profile created by the RF transmit pulse, slice select gradient, and rephasing gradient. The slice profile should be calibrated to quote the correct slice thickness and to ensure maximum rephasing. Also, when using fast imaging methods or 2-D or 3-D imaging, the shape of the profile can profoundly affect the results. As discussed earlier, there are many reasons why the RF profile does not match the desired result of a perfect boxcar profile. Fortunately, the RF slice select

Nulling Signal of a Slice, Slice Select Profile, and Radiofrequency Power profile can be measured by an experiment where the read direction is placed along the slice select axis. (The read gradient is discussed in Chapter B4.) 


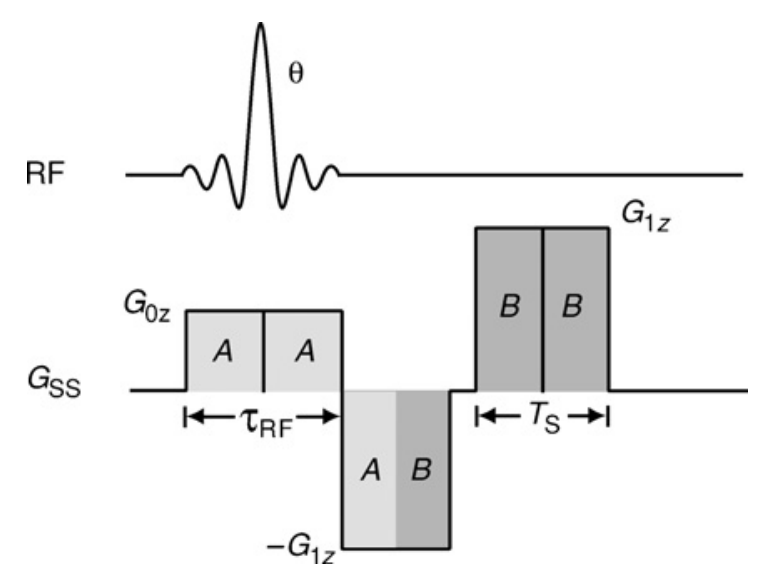

Figure B2.3.3 A sequence design for measuring the RF profile along the slice select direction. The slice select gradient $G_{0 z}$ is followed by a rephasing gradient $-G_{1 z}$. After an echo is formed, this gradient is kept constant and used to dephase the spins. Then the spins are rephased again by switching the polarity as shownin the figure. Another echo is formed during the sampling.

After the spins are rephased by a negative gradient followed by the RF pulse, a read gradient $G_{1 z}$ along the slice select direction can be placed as shown in Fig. B2.3.3 to generate an echo. From the Nyquist theorem (discussed in Chapter B4), the resolution of the slice profile is:

$$
\Delta s s=\frac{1}{\not G_{1 z} T_{\mathrm{s}}}
$$

where $T_{\mathrm{s}}$ is the sampling time. If the sequence has a low flip angle, then the RF bandwidth is related to the slice thickness through $B W_{\mathrm{RF}}=q G_{0 z} \mathrm{TH}$. The slice thickness is the number of collected sampling points $(N)$ multiplied by the resolution, i.e., $\mathrm{TH}=N \Delta s s$. If the RF pulse is a sinc function, then its zero crossing $n_{z c}$ is $B W_{\mathrm{RF}} \tau_{\mathrm{RF}}$ where $\tau_{\mathrm{RF}}$ is the time interval when the RF pulse is turned on. All these equations yield:

$$
N=n_{\mathrm{zc}} \frac{G_{1 z} T_{\mathrm{s}}}{G_{0 z} \tau_{\mathrm{RF}}}
$$

Thus, if $G_{1 z}$ is made large and $T_{\mathrm{s}}$ long, it is possible to acquire many points through the slice. To get a reasonable slice profile $N$ should be chosen to be 64 or greater.

\section{Defining slice thickness}

The term slice thickness is used to define the thickness of the region along the slice select direction which contributes signal to an MR image. The positions where the transverse magnetization is zero can be found from the solution of the Bloch equations (see UNIT B2.2). The slice thickness that we introduced through the RF bandwidth and the slice select gradient before was correct only when the RF field is a sinc function in time with a low flip angle. When the flip angle is small, the slice select profile in the frequency domain (and thus in the spatial domain) is simply the Fourier transformation of the RF field in the time domain. Figure B2.3.4 illustrates such a relation. A Gaussian pulse will have a Gaussian profile in the frequency (spatial) domain and an infinite sinc pulse will have a perfect boxcar profile.

Radiofrequency

Excitation and Reception

B2.3.5 
A

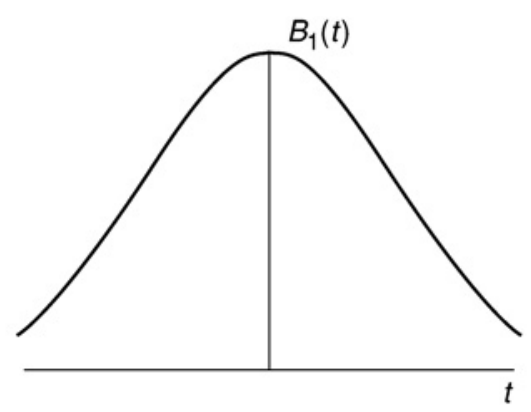

C

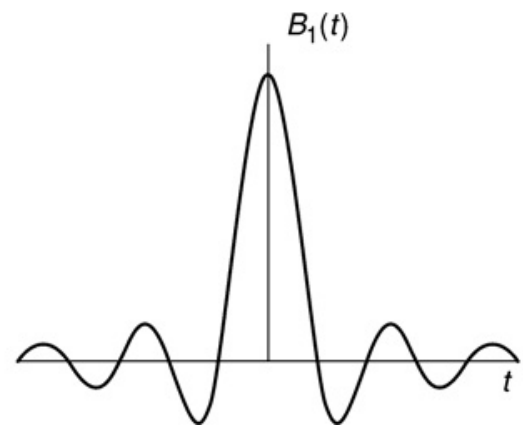

B

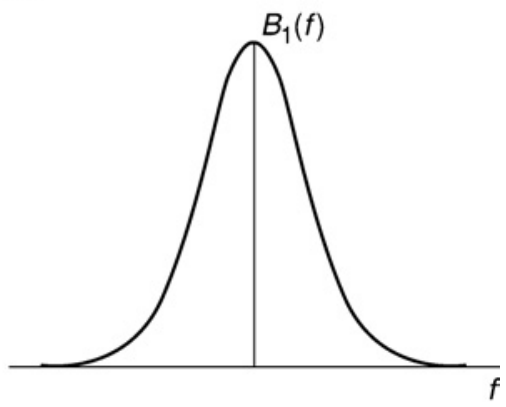

D

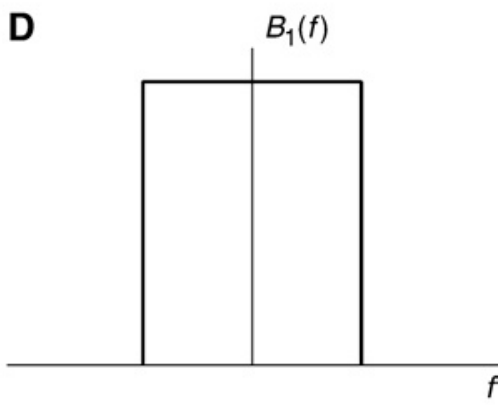

Figure B2.3.4 The graphs in (A) and (B) show a Gaussian pulse and its corresponding excitation profile. A sinc pulse appears in (C) and its corresponding excitation profile appears in (D). Alternatively, panel $\mathrm{D}$ can be used to represent a rect pulse, and panel $\mathrm{C}$ can be used to represent its excitation profile.

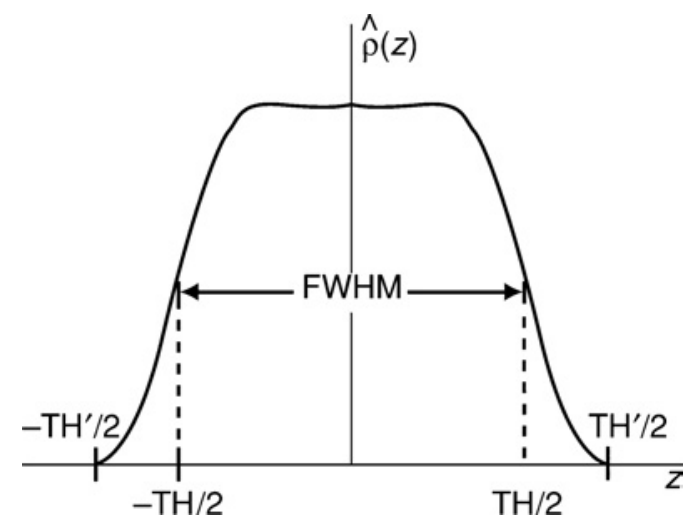

Figure B2.3.5 Illustration of the FWHM of a theoretical slice profile generated from an apodized sinc pulse. Although, $\mathrm{TH}=\mathrm{FWHM}$ is often chosen as the effective slice thickness, clearly information from outside this region is excited as well. Usually, excitation is well-behaved and no further excitation takes place outside the boundaries demarcated by $\pm \mathrm{TH}^{\prime} / 2$.

Nulling Signal of a Slice, Slice Select Profile, and Radiofrequency Power B2.3.6

Due to the finite time duration of the applied RF pulses, it is not possible to achieve a perfect boxcar slice profile; instead, the region of spins affected by the RF pulse goes smoothly to zero at the edges of the profile (see Fig. B2.3.5). As a result, it is necessary to define some criterion for quoting the slice thickness based upon the region where appreciable signal is contributed to the image. In general, the FWHM (full width at half maximum) of the measured slice select profile is used to define slice thickness as shown in Fig. B2.3.5. Similar to the use of the FWHM in defining resolution, it is 
necessary to keep in mind that this definition is somewhat arbitrary. For a slice whose profile approaches the ideal rectangular case, this definition is reasonable since very little transverse magnetization is created beyond the FWHM (see Fig. B2.3.5), and, therefore, the definition is accurate. If, instead, a Gaussian excitation is chosen, then there may be an appreciable amount of signal created outside of the FWHM of the image profile and, in this case, the FWTM (full width at tenth maximum) might be a better choice for slice thickness. It is important to remember that the thickness of the image profile is being discussed here. The FWHM of the applied RF pulse does not necessarily match the FWHM of the slice measured in the image. It is the image slice profile that is of interest. Consider two cases where this might be important, 3-D imaging and 2-D multislice imaging. If the $z$-direction field-of-view is chosen to be $\mathrm{TH}$ in a $3-\mathrm{D}$ experiment, then there may be a significant amount of aliasing in the image from the spins excited between $\mathrm{TH} / 2$ and $\mathrm{TH}^{\prime} / 2$ as labeled in Fig. B2.3.5. In a 2-D multislice experiment, if an adjacent slice is imaged right after the scan of the present slice, then there will be "crosstalk" between slices. Crosstalk refers to the situation where the excitation of one slice, significantly affects the signal from a neighboring slice. To avoid excessive crosstalk in 2-D multi-slice imaging, the distance between slice centers should be set to $\mathrm{TH}^{\prime}$ outside of which there is essentially no slice excitation.

\section{Radiative Power Deposition and Flip Angles of RF Pulses}

When dealing with RF pulses, there are several practical aspects of the RF pulse design that should be understood. First of all, it is useful to understand how much power must be supplied by the RF amplifiers in order to achieve a given flip angle in a given time for a specified set of slice parameters. A closely related issue of special interest for human imaging is the fact that some of the applied RF power will be deposited in the MRI sample as heat. In human imaging, strict guidelines are set for the SAR (Specific Absorption Rate) of RF power by the body. The present guidelines are that the power (or rather the power per unit mass, or W/kg) deposited should not exceed 1.5 to $3.0 \mathrm{~W} / \mathrm{kg}$ over the whole body, or 3 to $8 \mathrm{~W} / \mathrm{kg}$ "locally" (point by point). Therefore, it is important to understand the power and energy characteristics associated with RF pulses. In this section, several symmetric pulses with low flip angles applied during constant gradients are considered.

The energy density of an electromagnetic field is proportional to the magnitude squared of the magnetic (or the electric) field. Because the RF field is a function of time and rotates at the Larmor frequency $\omega_{0}$, the energy radiated from an RF field $B_{1}(\vec{r}, t)$ is proportional to the spatial and temporal integral of the energy density:

$$
W\left(\tau_{\mathrm{RF}}\right) \propto \omega_{0} \int_{0}^{\tau_{\mathrm{RF}}} d t \int d^{3} r B_{1}^{*}(\vec{r}, t) B_{1}(\vec{r}, t)
$$

The spatial dependence of $B_{1}$ is the same for each pulse, and, therefore, the spatial integral in Equation B2.3.9 is only a scale factor. The pertinent information for the MRI experiment is contained in the term:

$$
W_{p}=\int d t B_{1}(t) B_{1}^{*}(t)
$$

where the spatial dependence is neglected because the field is assumed to be uniform over the region of interest.

One aspect of the RF pulses that is difficult to determine analytically is the effect of truncating waveforms that are ideally infinite in time. Although these filter effects have been discussed at length, quantifying their effects is difficult. In general, the longer a

Radiofrequency Excitation and Reception

\section{B2.3.7}


waveform is, the closer it comes to producing the desired profile. Therefore, in what follows, it is assumed that a characteristic time can be defined for each pulse, and the time duration of the RF pulse is some multiple of this time so that the resulting slice profile is a reasonable approximation of the ideal case.

\section{Sinc pulse}

Consider generating a boxcar slice profile. This implies that a sinc pulse will be applied in the time domain. $B_{1}(t)$ is then:

$$
B_{1}(t)=B_{1} \operatorname{sinc}\left(\frac{\pi t}{\tau_{\text {sinc }}}\right)
$$

where $\tau_{\text {sinc }} \equiv 1 / B W_{\mathrm{RF}}$. The characteristic flip angle and radiative energy for this pulse must also be found. From Equation B2.3.10, the effective energy is equal to:

$$
W_{p}=B_{1}^{2} \int_{-\infty}^{\infty} \frac{\sin ^{2}\left(\frac{\pi t}{\tau_{\text {sinc }}}\right)}{\frac{\pi^{2} t^{2}}{\tau_{\text {sinc }}^{2}}} d t=B_{1}^{2} \tau_{\text {sinc }}
$$

where it is assumed that the pulse duration is sufficiently long that it can be approximated by the infinite time result. The central flip angle (see UNIT B2.2) associated with this pulse is:

$$
\theta_{0}=\gamma B_{1} \tau_{\text {sinc }}
$$

Alternately, the power can be expressed in terms of the flip angle and bandwidth by substituting $B_{1}$ as a function of $\theta_{0}$ (from Equation B2.3.13) into Equation :

$$
W_{p} \propto \theta_{0}^{2} / \tau_{\text {sinc }}=\theta_{0}^{2} B W_{\mathrm{RF}}
$$

It is obvious that if the flip angle doubles then the power will increase four times. On the other hand, if both the flip angle and the time duration of the RF pulse double, then the power will only increase by a factor of two.

Knowing the RF and imaging parameters is necessary for determining the energy used by the pulse, which is proportional to the energy deposited in the body. However, it is also important to understand what happens when these parameters are varied, to see what flexibility exists in slice select design (assuming TH and $\theta_{0}$ remain fixed). In order to minimize $T_{\mathrm{E}}$, for example, it might be desirable for a given experiment to minimize the time duration of the RF pulse. This will be accomplished if $\tau_{\mathrm{RF}}$ is fixed to be a multiple of $\tau_{\text {sinc }}$ and $\tau_{\text {sinc }}$ is reduced (i.e., the bandwidth of the RF pulse is increased). Recall that for a small flip angle, the slice thickness is:

$$
\mathrm{TH}=\frac{B W_{\mathrm{RF}}}{\not \mathrm{G}_{\mathrm{ss}}}=\frac{1}{\not \mathrm{G}_{\mathrm{ss}} \tau_{\mathrm{sinc}}}
$$

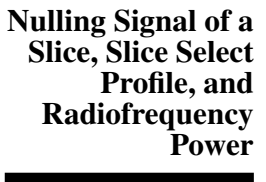

In this case, if:

$$
\tau_{\text {sinc }} \rightarrow \lambda \tau_{\text {sinc }}
$$


and $\theta_{0}$ and $\mathrm{TH}$ are kept constant, then:

$$
G_{\mathrm{ss}} \rightarrow \frac{G_{\mathrm{ss}}}{\lambda}, \quad B_{1} \rightarrow \frac{B_{1}}{\lambda}, \quad W_{p} \rightarrow \frac{W_{p}}{\lambda}
$$

If $\lambda$ equals $1 / 2$, for example, twice the slice select gradient, the $B_{1}$ amplitude, and the SAR value must all be physically available for the pulse to be used. Another crucial point is that four times as much RF power must be available from the RF amplifier, since:

$$
\text { power }=\frac{\text { energy }}{\text { time }} \propto \frac{W_{p}}{\tau_{\mathrm{RF}}}
$$

In general, there is an increase in power from the pulse of $1 / \lambda^{2}$. For this reason, very short time duration, larger flip angle pulses may be impossible to use because they require too much RF amplifier power. Of course, it is also possible to look at situations where other parameters are varied or fixed; the above is only an introduction to the topic.

\section{Simple harmonic pulse}

Another example is the finite-time, rect pulse. Consider a sinusoidal pulse on-resonance with $\omega_{0}$ and applied for a time $\tau_{\text {rect }}$ with constant amplitude. In the rotating frame:

$$
B_{1}(t)=B_{1} \operatorname{rect}\left(\frac{t}{\tau_{\text {rect }}}\right)
$$

The effective energy and the flip angle at the center of the slice are:

$$
W_{p}=B_{1}^{2} \tau_{\text {rect }}
$$

and

$$
\theta_{0}=\gamma B_{1} \tau_{\text {rect }}
$$

\section{Gaussian pulse}

The third example is a Gaussian pulse:

$$
B_{1}(t)=B_{1} e^{-t^{2} /\left(2 \tau_{\text {gauss }}^{2}\right)}
$$

This pulse decays to $e^{-1}$ of its maximum (at $t=0$ ) by the time $t=\sqrt{2} \tau_{\text {gauss. }}$ The energy and the central flip angle are now:

$$
W_{p}=B_{1}^{2} \int_{-\infty}^{\infty} d t e^{-t^{2} / \tau_{\text {gauss }}^{2}}=\sqrt{\pi} \tau_{\text {gauss }} B_{1}^{2}
$$

and

$$
\theta_{0}=\sqrt{2 \pi} \gamma B_{1} \tau_{\text {gauss }}
$$

respectively. The results derived for the three pulse envelopes are summarized in Figure B2.3.6. One can see that for the same flip angle at the center of the excited slice, the

Radiofrequency Excitation and Reception

B2.3.9 


\begin{tabular}{l|c|c} 
Pulse envelope, $B_{1}(t)$ & Flip angle & Effective energy \\
\hline$B_{1}$ rect $\left(\frac{t}{\tau_{\text {rect }}}\right)$ & $\gamma \mathrm{B}_{1} \tau_{\text {rect }}$ & $\mathrm{B}_{1}^{2} \tau_{\text {rect }}$ \\
\hline $\mathrm{B}_{1} \operatorname{sinc}\left(\frac{\pi \mathrm{t}}{\tau_{\text {sinc }}}\right)$ & $\gamma \mathrm{B}_{1} \tau_{\text {sinc }}$ & $\mathrm{B}_{1}^{2} \tau_{\text {sinc }}$ \\
\hline $\mathrm{B}_{1} \mathrm{e}^{-\mathrm{t} 2 /\left(2 \tau_{\text {gauss }}^{2}\right)}$ & $\sqrt{2 \pi} \gamma \mathrm{B}_{1} \tau_{\text {gauss }}$ & $\sqrt{\pi} \mathrm{B}_{1}^{2} \tau_{\text {gauss }}$ \\
\hline
\end{tabular}

Figure B2.3.6 Summary of pulse envelope properties.

Gaussian pulse has $\sqrt{2}$ less radiated energy that those from the sinc and harmonic pulses (but outside the center the flip angle varies spatially).

\section{KEY REFERENCE}

Haacke, E.M., Brown, R.W., Thompson, M.R., and Venkatesan, R. 1999. Magnetic Resonance Imaging: Physical Principles and Sequence Design. John Wiley and Sons, New York.

This text covers the technical aspects presented here, but in more detail, and also discusses more advanced materials.

Contributed by Yu-Chung Norman Cheng and E. Mark Haacke

Case Western Reserve University

Cleveland Ohio

and The MRI Institute for Biomedical Research

Detroit, Michigan

Nulling Signal of a Slice, Slice Select

Profile, and

Radiofrequency

Power

B2.3.10 